\title{
Almost Periodic Solutions for Some Convolution Equations
}

\author{
Silvia-Otilia Corduneanu
}

\begin{abstract}
We use the theory of Fourier series for almost periodic functions to looking for complex-valued functions $f$ which are almost periodic on $\mathbb{R}$ and satisfy the following equation

$$
f(x)=\lim _{t \rightarrow \infty} \frac{1}{2 t} \int_{-t}^{t}\left[\int_{-\infty}^{+\infty} f(x-y-z) \varphi(z) d z\right] g(y) d y+h(x), \quad x \in \mathbb{R} .
$$

In this context $g$ and $h$ are almost periodic functions on $\mathbb{R}$ and $\varphi$ belongs to $L^{1}(\mathbb{R})$.
\end{abstract}

\section{Introduction}

Let $\widehat{\mathbb{R}}$ be the dual group of the group $\mathbb{R}$ and $A P(\mathbb{R})$ the space of all almost periodic complex-valued functions defined on $\mathbb{R}$. We denote by $M(f)$ or $M_{x}[f(x)]$ the mean of an almost periodic function $f$ and by $c_{\gamma}(f)$ the Fourier coefficient of $f$ corresponding to a character $\gamma \in \widehat{\mathbb{R}}$. If the functions $f$ and $g$ belong to $A P(\mathbb{R})$ and $\varphi \in L^{1}(\mathbb{R})$, then the function

$$
\Phi(x)=M_{y}[(\varphi * f)(x-y) g(y)], \quad x \in \mathbb{R},
$$

is an almost periodic function. Consider $\gamma \in \widehat{\mathbb{R}}, f \in A P(\mathbb{R}), g \in A P(\mathbb{R})$ and $\varphi \in L^{1}(\mathbb{R})$. We calculate the Fourier coefficient of $\Phi$ corresponding to the character $\gamma$, more precisely we obtain:

$$
c_{\gamma}(\Phi)=c_{\gamma}(f) c_{\gamma}(g) \widehat{\varphi}(\gamma),
$$

Key Words: Almost periodic function, convolution equation, Fourier coefficients.

2010 Mathematics Subject Classification: Primary 42A05,42A10; Secondary 39B32.

Received: February, 2013.

Revised: February, 2013

Accepted: April, 2013. 
where $\widehat{\varphi}$ is the the Fourier transform of $\varphi$. In this paper we consider the following equation

$$
f(x)=M_{y}[(\varphi * f)(x-y) g(y)]+h(x), \quad x \in \mathbb{R},
$$

where $g \in A P(\mathbb{R}), h \in A P(\mathbb{R})$ and $\varphi \in L^{1}(\mathbb{R})$. If we use the formula of the mean we can rewrite equation (1.2) as

$$
f(x)=\lim _{t \rightarrow \infty} \frac{1}{2 t} \int_{-t}^{t}\left[\int_{-\infty}^{+\infty} f(x-y-z) \varphi(z) d z\right] g(y) d y+h(x),
$$

where $x \in \mathbb{R}$. For solving equation (1.2) we use the theory of Fourier series for almost periodic functions. Taking into account the property that two almost periodic functions coincide if they have the same Fourier coefficients we establish an existence and uniqueness result in the case of equation (1.2). Finally we establish an existence and uniqueness result in the case of equation

$$
f(x)=M_{y}[(\varphi * f)(x-y) g(y)]+\lim _{t \rightarrow \infty} \frac{1}{2 t} \int_{-t}^{t} k(x-y) l(y) d y,
$$

where $x \in \mathbb{R}$,

$$
M_{y}[(\varphi * f)(x-y) g(y)]=\lim _{t \rightarrow \infty} \frac{1}{2 t} \int_{-t}^{t}\left[\int_{-\infty}^{\infty} f(x-y-z) \varphi(z) d z\right] g(y) d y,
$$

for every $x \in \mathbb{R}$ and the functions $k$ and $l$ belong to $A P(\mathbb{R})$.

\section{Preliminaries}

Consider $\mathcal{C}(\mathbb{R})$ the set of all bounded continuous complex-valued functions on $\mathbb{R}$ and denote by $\|\cdot\|$ the supremum norm defined on $\mathcal{C}(\mathbb{R})$. Let $\lambda$ be the Lebesgue measure on $\mathbb{R}$. The space of Lebesgue measurable functions $f$ on $\mathbb{R}$, with $\int_{\mathbb{R}}|f(x)| d \lambda(x)<\infty$ will be denoted by $L^{1}(\mathbb{R})$, and the norm of $L^{1}(\mathbb{R})$, by $\|\cdot\|_{1}$. We use $\widehat{\mathbb{R}}$ to denote the dual group of the group $\mathbb{R}$ and $[\widehat{\mathbb{R}}]$ the space of all trigonometric polynomials on $\mathbb{R}$. If $\varphi \in L^{1}(\mathbb{R})$, the Fourier transform of $\varphi$, denoted by $\widehat{\varphi}$, is given by

$$
\widehat{\varphi}(\gamma)=\int_{\mathbb{R}} \varphi(x) \bar{\gamma}(x) d x, \quad \gamma \in \widehat{\mathbb{R}}
$$

For $f \in \mathcal{C}(\mathbb{R})$ and $a \in \mathbb{R}$, the translate of $f$ by $a$ is $f_{a}(x)=f(x+a)$ for all $x \in \mathbb{R}$. We remind the definition of an almost periodic function which can be found in [4], [5], [11], [13], [14]. 
Definition 2.1. A function $f \in \mathcal{C}(\mathbb{R})$ is called an almost periodic function on $\mathbb{R}$, if the family of translates of $f,\left\{f_{a} \mid a \in \mathbb{R}\right\}$ is relatively compact in the sense of uniform convergence on $\mathbb{R}$.

The space $A P(\mathbb{R})$ is a Banach algebra with respect to the supremum norm and $[\widehat{\mathbb{R}}]$ is dense in $A P(\mathbb{R})$ in the sense of uniform convergence. There exists a unique positive linear functional $M: A P(\mathbb{R}) \rightarrow \mathbb{C}$ such that $M\left(f_{a}\right)=M(f)$, for all $a \in \mathbb{R}, f \in A P(\mathbb{R})$ and $M(\mathbf{1})=1$. (In this context we denote by $\mathbf{1}$ the constant function which is 1 for all $x \in \mathbb{R})$. If $f \in A P(\mathbb{R})$ we define the mean of $f$ as being the above complex number $M(f)$, we put $c_{\gamma}(f)=M(\bar{\gamma} f)$ for all $\gamma \in \widehat{\mathbb{R}}$ and we call $c_{\gamma}(f)$, the Fourier coefficient of $f$ corresponding to $\gamma \in \widehat{\mathbb{R}}$. If $f \in A P(\mathbb{R})$ the set $\{\gamma \in \widehat{\mathbb{R}} \mid M(\bar{\gamma} f) \neq 0\}$ is at most a countable set (see Theorem 1.15 from [5]) and we denote it by $\left\{\gamma_{n} \in \widehat{\mathbb{R}} \mid n \in \mathbb{N}\right\}$. The Fourier series of $f$ is

$$
\sum_{n=1}^{\infty} c_{\gamma_{n}}(f) \gamma_{n}
$$

If $\varphi \in L^{1}(\mathbb{R})$ and $f \in A P(\mathbb{R})$, their convolution $\varphi * f$ belongs to $A P(\mathbb{R})$. We recall that

$$
\varphi * f(x)=\int_{\mathbb{R}} f(x-y) \varphi(y) d y, \quad x \in \mathbb{R} .
$$

\section{Convolution equations with almost periodic solutions}

Theorem 3.1. If $g$ and $f$ belong to $A P(\mathbb{R})$ and $\varphi$ belongs to $L^{1}(\mathbb{R})$ then the function $\Phi: \mathbb{R} \rightarrow \mathbb{C}$ given by

$$
\Phi(x)=M_{y}[(\varphi * f)(x-y) g(y)], \quad x \in \mathbb{R},
$$

is almost periodic and for every $\gamma \in \widehat{\mathbb{R}}$, the following equality is true

$$
c_{\gamma}(\Phi)=c_{\gamma}(f) c_{\gamma}(g) \widehat{\varphi}(\gamma) .
$$

Proof. We first check the conclusion of the theorem for characters of $\widehat{\mathbb{R}}$, after that for trigonometric polynomials of $[\widehat{\mathbb{R}}]$ and finally for arbitrary almost periodic functions. Consider $\gamma_{0} \in \widehat{\mathbb{R}}$, suppose that $f=\gamma_{0}$ and denote $\Phi_{0}(x)=M_{y}\left[\left(\varphi * \gamma_{0}\right)(x-y) g(y)\right], x \in \mathbb{R}$. With this choice we calculate $\varphi * \gamma_{0}$ as

$$
\varphi * \gamma_{0}(x)=\int_{-\infty}^{\infty} \gamma_{0}(x-y) \varphi(y)=\widehat{\varphi}\left(\gamma_{0}\right) \gamma_{0}(x), \quad x \in \mathbb{R} .
$$

We observe that

$$
\Phi_{0}(x)=M_{y}\left[\left(\varphi * \gamma_{0}\right)(x-y) g(y)\right]=\widehat{\varphi}\left(\gamma_{0}\right) c_{\gamma_{0}}(g) \gamma_{0}(x), \quad x \in \mathbb{R}
$$


therefore, if $f=\gamma_{0} \in \widehat{\mathbb{R}}$, then $\Phi_{0} \in A P(\mathbb{R})$. On the other hand we get, for $\gamma \in \widehat{\mathbb{R}}$

$$
c_{\gamma}(f)=M\left(\bar{\gamma} \gamma_{0}\right)= \begin{cases}1, & \gamma=\gamma_{0} \\ 0, & \gamma \neq \gamma_{0}\end{cases}
$$

hence

$$
c_{\gamma}\left(\Phi_{0}\right)=c_{\gamma}(f) c_{\gamma}(g) \widehat{\varphi}(\gamma)
$$

It immediately follows, that for $f \in[\widehat{\mathbb{R}}]$, the function $\Phi$ which is given in relation (3.1) is an almost periodic function on $\mathbb{R}$ and relation (3.2) is valid. Suppose that $f \in A P(\mathbb{R})$ and is arbitrary. There exists a sequence $\left(f_{n}\right)_{n}$ such that

$$
\left((\forall n \in \mathbb{N})\left(f_{n} \in[\widehat{\mathbb{R}}]\right)\right) \wedge\left(\left\|f_{n}-f\right\| \rightarrow 0\right) .
$$

If we denote

$$
\Phi_{n}(x)=M_{y}\left[\left(\varphi * f_{n}\right)(x-y) g(y)\right], \quad x \in \mathbb{R}, n \in \mathbb{N},
$$

from the previous part it results that $(\forall n \in \mathbb{N})\left(\Phi_{n} \in A P(\mathbb{R})\right)$. Taking into account that for every $n \in \mathbb{N}$ and $x \in \mathbb{R}$

$$
\begin{aligned}
& \left|\Phi_{n}(x)-\Phi(x)\right| \\
& =\left|M_{y}\left[\left(\varphi * f_{n}\right)(x-y) g(y)\right]-M_{y}[(\varphi * f)(x-y) g(y)]\right| \\
& \leq \lambda(|\varphi|) M(|g|)\left\|f_{n}-f\right\|,
\end{aligned}
$$

it follows that $\Phi_{n} \rightarrow \Phi$ uniformly, hence $\Phi \in A P(\mathbb{R})$. We see also from the previous part that for $\gamma \in \widehat{\mathbb{R}}$,

$$
(\forall n \in \mathbb{N})\left(c_{\gamma}\left(\Phi_{n}\right)=c_{\gamma}\left(f_{n}\right) c_{\gamma}(g) \widehat{\varphi}(\gamma)\right) .
$$

Using the fact that $\Phi_{n} \rightarrow \Phi$ uniformly it results that $c_{\gamma}\left(\Phi_{n}\right) \rightarrow c_{\gamma}(\Phi)$ and further, for $\gamma \in \widehat{\mathbb{R}}$,

$$
c_{\gamma}(\Phi)=c_{\gamma}(f) c_{\gamma}(g) \widehat{\varphi}(\gamma)
$$

Consider $g \in A P(\mathbb{R}), f \in A P(\mathbb{R})$ and $\varphi \in L^{1}(\mathbb{R})$. Suppose that the function $h \in A P(\mathbb{R})$ has the Fourier series

$$
\sum_{n=1}^{\infty} c_{\gamma_{n}}(h) \gamma_{n} .
$$


In what follows we treat equation

$$
f(x)=M_{y}[(\varphi * f)(x-y) g(y)]+h(x), \quad x \in \mathbb{R}
$$

Using the formula of the mean we can rewrite equation (3.3) as

$$
f(x)=\lim _{t \rightarrow \infty} \frac{1}{2 t} \int_{-t}^{t}\left[\int_{-\infty}^{+\infty} f(x-y-z) \varphi(z) d z\right] g(y) d y+h(x),
$$

where $x \in \mathbb{R}$. Taking into account the property that two almost periodic functions coincide if they have the same Fourier coefficients, we establish an existence and uniqueness result in the case of equation (3.3).

Theorem 3.2. Consider $g \in A P(\mathbb{R}), f \in A P(\mathbb{R})$ and $\varphi \in L^{1}(\mathbb{R})$. If $\sum_{n=1}^{\infty}\left|c_{\gamma_{n}}(h)\right|<\infty$ and $\inf _{\gamma \in \widehat{\mathbb{R}}}\left|1-c_{\gamma}(g) \widehat{\varphi}(\gamma)\right| \in(0, \infty)$, then equation (3.3) has a unique solution $f \in A P(\mathbb{R})$.

Proof. Let us denote

$$
\delta=\inf _{\gamma \in \widehat{\mathbb{R}}}\left|1-c_{\gamma}(g) \widehat{\varphi}(\gamma)\right|
$$

and consider $\gamma_{0} \in \widehat{\mathbb{R}}$. Taking into account the conclusion of Theorem 3.1 we deduce that if $f \in A P(\mathbb{R})$ would be solution of the equation (3.3) then

$$
c_{\gamma_{0}}(f)=c_{\gamma_{0}}(f) c_{\gamma_{0}}(g) \widehat{\varphi}\left(\gamma_{0}\right)+c_{\gamma_{0}}(h)
$$

Therefore we consider the series

$$
\sum_{n=1}^{\infty} \frac{c_{\gamma_{n}}(h)}{1-\widehat{\varphi}\left(\gamma_{n}\right) c_{\gamma_{n}}(g)} \gamma_{n}
$$

which is uniformly convergent, because

$$
\left|\frac{c_{\gamma_{n}}(h)}{1-\widehat{\varphi}\left(\gamma_{n}\right) c_{\gamma_{n}}(g)} \gamma_{n}(x)\right| \leq \frac{1}{\delta}\left|c_{\gamma_{n}}(h)\right|, \quad x \in \mathbb{R}
$$

We denote by $f$ the sum of the series (3.5). It is obvious that $f$ is an almost periodic function. Based on the property that two almost periodic functions coincide if they have the same Fourier series we conclude that $f$ is the unique solution of the equation (3.3). 
Consider $\varphi \in L^{1}(\mathbb{R}), g \in A P(\mathbb{R}), k \in A P(\mathbb{R})$ and $l \in A P(\mathbb{R})$. Next we treat equation

$$
f(x)=M_{y}[(\varphi * f)(x-y) g(y)]+\lim _{t \rightarrow \infty} \frac{1}{2 t} \int_{-t}^{t} k(x-y) l(y) d y,
$$

where $x \in \mathbb{R}$. Equation (3.6) is a a particular case of equation (3.3).

Theorem 3.3. If in the previous context we consider that $\|\varphi\|_{1}\|g\| \in(0,1)$ then equation (3.6) has a unique solution $f \in A P(\mathbb{R})$.

Proof. Let us denote

$$
h(x)=\lim _{t \rightarrow \infty} \frac{1}{2 t} \int_{-t}^{t} k(x-y) l(y) d y, \quad x \in \mathbb{R} .
$$

Taking into account that $h(x)=M_{y}[k(x-y) l(y)], x \in \mathbb{R}$, we get the equality

$$
c_{\gamma}(h)=c_{\gamma}(k) \cdot c_{\gamma}(l), \quad \gamma \in \widehat{\mathbb{R}} .
$$

We observe that equation (3.6) is a a particular case of equation (3.3) so we check the first hypothesis of Theorem 3.2. Using a Cauchy inequality we obtain

$$
\sum_{n=1}^{\infty}\left|c_{\gamma_{n}}(h)\right|=\sum_{n=1}^{\infty}\left|c_{\gamma_{n}}(k)\right|\left|c_{\gamma}(l)\right| \leq\left[\sum_{n=1}^{\infty}\left|c_{\gamma_{n}}(k)\right|^{2}\right]^{\frac{1}{2}}\left[\sum_{n=1}^{\infty}\left|c_{\gamma}(l)\right|^{2}\right]^{\frac{1}{2}} .
$$

The functions $k$ and $l$ are almost periodic on $\mathbb{R}$, hence their Fourier series satisfy the Parseval equality, i.e.,

$$
\sum_{n=1}^{\infty}\left|c_{\gamma_{n}}(k)\right|^{2}=M\left(|k|^{2}\right)
$$

and

$$
\sum_{n=1}^{\infty}\left|c_{\gamma_{n}}(l)\right|^{2}=M\left(|l|^{2}\right)
$$

On the other hand taking into account that

$$
\left|c_{\gamma}(g) \widehat{\varphi}(\gamma)\right| \leq\|g\| \cdot\|\varphi\|_{1}, \quad \gamma \in \widehat{\mathbb{R}}
$$

we obtain

$$
\inf _{\gamma \in \widehat{\mathbb{R}}}\left|1-c_{\gamma}(g) \widehat{\varphi}(\gamma)\right| \geq \delta,
$$

where $\delta=1-\|g\| \cdot\|\varphi\|_{1}>0$. We can apply Theorem 3.2, and we conclude that equation (3.6) has a unique solution $f \in A P(\mathbb{R})$. 


\section{References}

[1] Ait Dads, E., Ezzinbi, K.: Existence of positive pseudo almost periodic solution for a class of functional equations arising in epidemic problems, Cybernet. Systems Anal., 30 (1994), No. 6 , 900-910.

[2] Ait Dads, E., Cieutat, P., Lhachimi, L.: Existence of positive almost periodic or ergodic solutions for some neutral nonlinear integral equations, Differential and Integral Equations, 22 (2009), No. 11-12, 1075-1096.

[3] Argabright, L.N., Gil de Lamadrid, J.: Fourier Analysis of Unbounded Measures on Locally Compact Abelian Groups, Mem. Amer. Math. Soc. 145 (1974).

[4] Argabright, L.N., Gil de Lamadrid, J: Almost Periodic Measures, Mem. Amer. Math. Soc. 428 (1990).

[5] Corduneanu, C.: Almost Periodic Functions, Interscience Publishers, New York, London, Sydney, Toronto, 1968.

[6] Corduneanu, S.O.: Inequalities for Almost Periodic Measures, Mathematical Inequalities \& Applications, Volume 5, No. 1 (2002), 105 - 111.

[7] Corduneanu, S.O.: Inequalities for a Class of Means with Parameter, Buletinul Institutului Politehnic din Iaşi, Tomul LIII (LVII), Fasc. 5, 2007, $77-84$.

[8] Corduneanu, S.O.: Equations Involving the Mean of Almost Periodic Measures, Operator Theory: Advances and Applications, 201 (2009), Birkhuser Verlag Basel/Switzerland, 125-133.

[9] Corduneanu, S.O.: Positive Almost Periodic Solutions of Some Convolution Equations, Positivity 14 (2010), DOI 10.1007/s11117-010-0081-9, 623-636.

[10] Deimling, K.: Nonlinear analysis, Springer-Verlag, Berlin, 1985.

[11] Eberlein, W.F.: Abstract Ergodic Theorems and Weak Almost Periodic Functions, Trans Amer. Math. Soc. 67(1949), 217-240.

[12] Ezzinbi, K., Hachimi, M.A.: Existence of positive almost periodic solutions of functional equations via Hilbert's projective metric, Nonlinear Anal., 26 (1996), 1169-1176.

[13] Gil de Lamadrid, J.: Sur les Mesures Presque Périodiques, Astérisque 4, 1973, 61-89. 
[14] Hewitt, E., Ross, K.A.: Abstract Harmonic Analysis Vol. I, Springer Verlag, Berlin, Göttingen, Heidelberg, 1963

[15] Rudin, W.: Fourier Analysis on Groups, Interscience Tracts in Pure and Applied Mathematics, Number 12, Interscience Publishers - John Wiley and Sons, New York, London, 1962.

Silvia-Otilia CORDUNEANU,

Department of Mathematics,

Gh. Asachi Technical University of Iaşi,

Bdul Carol I 11, 700506 Iaşi, Romania.

Email: scorduneanu@yahoo.com 